\title{
Magnetic extraction of energy from accretion disc around a rotating black hole*
}

\author{
GONG Xiao-Long, WANG Ding-Xiong ${ }^{\dagger}$, and YE Yong-Chun \\ Department of Physics, Huazhong University of Science and Technology, Wuhan 430074
}

April 18, 2004

\begin{abstract}
An analytical expression for the disc power is derived based on an equivalent circuit in black hole (BH) magnetosphere with a mapping relation between the radial coordinate of the disc and that of unknown astrophysical load. It turns out that this disc power is comparable with two other disc powers derived in the Poynting flux and hydrodynamic regimes, respectively. In addition, the relative importance of the disc power relative to the BZ power is discussed. It is shown that the BZ power is generally dominated by the disc power except some extreme cases. Furthermore, we show that the disc power derived in our model can be well fitted with the jet power of M87.
\end{abstract}

PACS numbers: 97.60.Lf, 98.62.Mw, 98.62.Js

As is well known, jets exist in many astronomical cases, such as active galactic nuclei, quasars, and young stellar objects. The association of jets with magnetized accretion discs or magnetized central objects (black holes or stars) is strongly supported by the recent observations of Hubble Space Telescope (HST), Chandra, and VLBI. ${ }^{[1,2]}$ Different theoretical models have been proposed for acceleration and collimation of jets, which can be divided into two main regimes, the Poynting flux regime and the hydromagnetic regime ${ }^{[3-6]}$. Both regimes are related to a poloidal magnetic field threading the disc, from which energy and angular momentum are extracted. In the Poynting flux regime, energy is extracted in Poynting flux (i.e. purely electromagnetic energy), but in the form of magnetically driven material winds in the latter regime.

Recently, Cao discussed the disc power in the hydrodynamic regime, and found that the disc power (henceforth $P_{M H D}$ ) is mainly determined by the strength and the configuration of the poloidal magnetic field. ${ }^{[7]}$ Lee discussed the disc power in the Poynting flux regime (henceforth $P_{E M}^{I}$ ), and found that the Poynting flux caused by a rotating magnetic field with Keplerian angular velocity can balance the energy and angular momentum conservation of a stationary accretion flow. ${ }^{[8]}$ In this Letter, we derive the disc power (henceforth $P_{E M}^{I I}$ ) by using an equivalent circuit in black hole $(\mathrm{BH})$ magnetosphere, and compare $P_{E M}^{I I}$ with $P_{M H D}$ and $P_{E M}^{I}$, respectively. Throughout this Letter the geometric units $G=c=1$ are used.

In order to derive the expression of the disc power $P_{E M}^{I I}$ we make the following assumptions:

1. The disc is thin, Keplerian, stable and perfectly conducting, which lies in the equatorial plane of the $\mathrm{BH}$ with the inner boundary being at the marginally stable orbit with the radius $r_{i n}=r_{m s}{ }^{[9]}$

2. The configuration of the poloidal magnetic field of the BH-disc system is shown in Fig. 1. The poloidal magnetic field lines thread both the $\mathrm{BH}$ horizon and the surrounding disc, connecting the disc with the remote astrophysical load. The shape of each field line threading the disc at radius $r$ has an analogy with that in the asymptotic jet model. ${ }^{[10]}$ It is assumed that the asymptotic radius of each field line $r^{\prime}$ is smaller than its light radius $R_{L}$. The asymptotic radius $r^{\prime}$ is related to the disc radius $r$ by

$$
r^{\prime}=\lambda r
$$

3. The astrophysical load is axisymmetric, being located evenly in a plane $\boldsymbol{P}$ with some height above the disc. The surface resistivity $\sigma_{L}$ of the load obeys the following relation, $\sigma_{L}=\alpha_{Z} \sigma_{H}=4 \pi \alpha_{Z}$, where $\sigma_{H}=4 \pi=377 \mathrm{ohm}$ is the surface resistivity of the $\mathrm{BH}$ horizon, and $\alpha_{Z}=1$ is taken in calculations.

4. The poloidal magnetic field on the disc varies as a power law as given in Refs.[11-13],

$$
B_{D}^{p} \propto \xi^{-n},
$$

\footnotetext{
* Supported by the National Natural Science Foundation of China under Grant Nos 10173004, 10373006 and 10121503

$\dagger$ To whom correspondence should be addressed. Email: dxwang@hust.edu.cn
} 


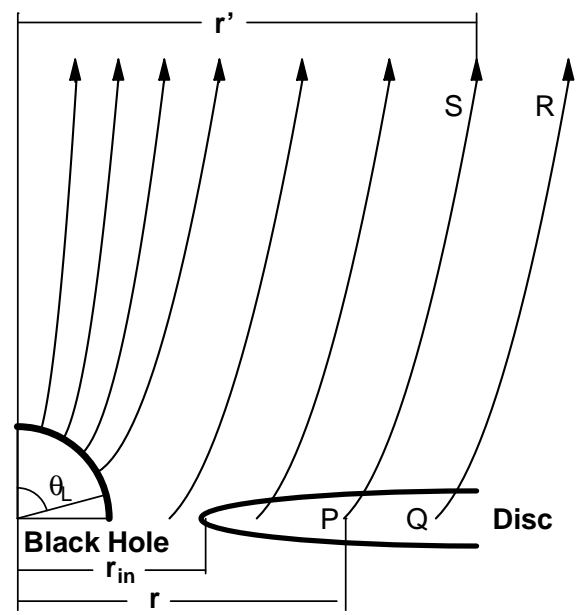

FIG. 1: Configuration of poloidal magnetic field threading the BH and its surrounding disc.

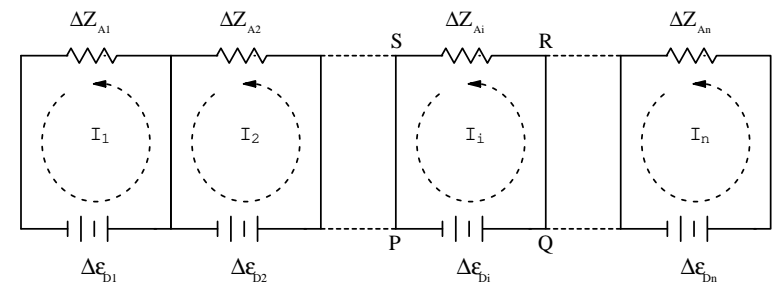

FIG. 2: Equivalent circuit for magnetic extraction of energy from accretion disc around a rotating BH.

where $\xi \equiv r / r_{m s}$ is the radial parameter of the disc defined in terms of the radius of inner edge of the disc. The field threading the $\mathrm{BH}$ is just a continuation of the field threading the disc, and the following relation is satisfied, ${ }^{[7]}$

$$
B_{H}^{p}=\varsigma B_{D}^{p}\left(r_{m s}\right),
$$

where $B_{H}^{p}$ and $B_{D}^{p}\left(r_{m s}\right)$ are the magnetic fields at the horizon and the inner edge of the disc, respectively. Some authors ${ }^{[14,15]}$ argued that the magnetic field threading the BH should not be significantly stronger than the field threading the inner disc. In this Letter $\varsigma=3$ is assumed in calculations.

Based on MacDonald and Thorne's work, ${ }^{[16]}$ we propose an equivalent circuit to calculate the disc power as shown in Fig.2. The segments $\boldsymbol{P} \boldsymbol{S}$ and $\boldsymbol{Q R}$ represent two adjacent magnetic surfaces consisting of the field lines connecting the disc and the load, and the segments $\boldsymbol{P} \boldsymbol{Q}$ and $\boldsymbol{R} \boldsymbol{S}$ represent the disc surface and the load sandwiched by the two surfaces, respectively. The quantities $\Delta Z_{A}$ and $\Delta \varepsilon_{D}$ (the subscript " $i "$ is omitted) are the resistance of the load and $\boldsymbol{e} \boldsymbol{m} \boldsymbol{f}$ due to the rotation of the disc, respectively. The disc load is neglected in calculations due to its perfect conductivity.

Based on assumptions (11) and (2) the load resistance $\Delta Z_{A}$ between the two adjacent magnetic surfaces can be written as

$$
\Delta Z_{A}=\sigma_{L} \frac{d r^{\prime}}{2 \pi r^{\prime}}=2 \frac{d r}{r} .
$$


The following equations are used in deriving disc power, which are almost the same as given in deriving the $\mathrm{BZ}$ power and $\mathrm{MC}$ power in our previous work. ${ }^{[12,13]}$

$$
\Delta P_{E M}^{I I}=\left(I^{p}\right)^{2} \Delta Z_{A}, \quad I^{p}=\Delta \varepsilon_{D} / \Delta Z_{A}, \quad \Delta \varepsilon_{D}=\left(\Delta \Psi_{D} / 2 \pi\right) \Omega_{D},
$$

where $I^{p}$ is the current in each loop, and $\Delta \Psi_{D}$ is the magnetic flux between the two adjacent magnetic surfaces,

$$
\Delta \Psi_{D}=B_{D}^{p} 2 \pi(\varpi \rho / \sqrt{\Delta})_{\theta=\pi / 2} d r
$$

The quantity $\Omega_{D}$ is the angular velocity of the disc at the place where the magnetic flux penetrates, and it reads

$$
\Omega_{D}=\frac{1}{M\left(\xi^{3 / 2} \chi_{m s}^{3}+a_{*}\right)}
$$

The concerned Kerr metric coefficients are given as follows, ${ }^{[17]}$

$$
\left\{\begin{array}{l}
\varpi=(\Sigma / \rho) \sin \theta \\
\Sigma^{2} \equiv\left(r^{2}+a^{2}\right)^{2}-a^{2} \Delta \sin ^{2} \theta \\
\rho^{2} \equiv r^{2}+a^{2} \cos ^{2} \theta \\
\Delta \equiv r^{2}+a^{2}-2 M r
\end{array}\right.
$$

Combining Eqs.(2) with (9), we have the poloidal magnetic field on the disc expressed by

$$
B_{D}^{p}=\varsigma^{-1} B_{H}^{p} \xi^{-n}
$$

Incorporating Eqs. (5) - (9), we have

$$
\Delta P_{E M}^{I I} / P_{0}=f\left(a_{*}, \xi, n\right) d \xi
$$

where the function $f\left(a_{*}, \xi, n\right)$ is expressed by

$$
f\left(a_{*}, \xi, n\right)=\frac{\chi_{m s}^{8}\left(1+\xi^{-2} \chi_{m s}^{-4} a_{*}^{2}+2 a_{*}^{2} \xi^{-3} \chi_{m s}^{-6}\right) \xi^{-2 n+3}}{2 \alpha_{Z} \varsigma^{2}\left(1+\xi^{-2} \chi_{m s}^{-4} a_{*}^{2}-2 \xi^{-1} \chi_{m s}^{-2}\right)\left(\xi^{3 / 2} \chi_{m s}^{3}+a_{*}\right)^{2}} .
$$

The quantity $P_{0}$ is defined as

$$
P_{0}=B_{H}^{2} M^{2} \approx B_{4}^{2}\left(M / M_{\odot}\right)^{2} \times 6.59 \times 10^{28} \mathrm{erg} \cdot \mathrm{s}^{-1},
$$

where $B_{4}$ and $M / M_{\odot}$ are the strength of $B_{H}^{p}$ and the $\mathrm{BH}$ mass in the units of $10^{4}$ gauss and one solar mass, respectively. Integrating Eq.(10) over the radial parameter $\xi$, we have the expression for the disc power as follows,

$$
P_{E M}^{I I}\left(a_{*}, \xi, n\right) / P_{0}=\int_{1}^{\xi} f\left(a_{*}, \xi^{\prime}, n\right) d \xi^{\prime}
$$

Cao derived the disc power in the hydromagnetic regime as follows, ${ }^{[7]}$ 


$$
\begin{gathered}
L_{d}=\frac{1}{2} \int\left(B_{D}^{p}\right)^{2}\left[r \Omega_{D}(r)\right]^{\alpha} f\left(\alpha, \gamma_{j}\right) r d r \\
f\left(\alpha, \gamma_{j}\right)=\left(\gamma_{j}-1\right) \gamma_{j}^{\alpha}\left(\gamma_{j}^{2}-1\right)^{-(\alpha+1) / 2},
\end{gathered}
$$

where the function $f\left(\alpha, \gamma_{j}\right)$ depends on the self-similar index $\alpha>1$ and the Lorentz factor of the jet $\gamma_{j}$. Since both $r \Omega_{D}(r)$ and $f\left(\alpha, \gamma_{j}\right)$ are always less than unity, the upper limit to the disc power $L_{d}$ can be written as

$$
L_{d}<P_{M H D}=\frac{1}{2} \int\left(B_{D}^{p}\right)^{2} r^{2} \Omega_{D}(r) d r .
$$

The expression of the poloidal magnetic field is expressed as follows, ${ }^{[8]}$

$$
B_{D}^{p}(r) \propto(r / M)^{-3 / 4}\left(M / M_{\odot}\right)^{-1 / 2} A^{-1} B E^{1 / 2},
$$

where $A, B$ and $E$ are general relativistic correction factors given in Ref.[9].Combining Eq.(16) with Eq.(17), we obtain

$$
P_{M H D}\left(a_{*}, \xi\right) / P_{0}=\frac{1}{2 \varsigma^{2}} \int_{1}^{\xi} \frac{\chi_{m s}^{3} \xi^{\prime-1}}{K^{2}\left(1+\xi^{\prime-3 / 2} \chi_{m s}^{-3} a_{*}\right)} d \xi^{\prime}
$$

where $\mathrm{K}=\left(A^{-1} B E^{1 / 2}\right)_{r_{i n}} /\left(A^{-1} B E^{1 / 2}\right)_{r}$.

Lee derived the disc power $P_{E M}^{I}$ in the Poynting flux regime as follows, ${ }^{[8]}$

$$
P_{E M}^{I}=4\left\{\left[-u_{0}\left(r_{\text {out }}\right)\right]-\left[-u_{0}\left(r_{\text {in }}\right)\right]\right\}\left[B_{D}^{p}\left(r_{\text {in }}\right) r_{\text {in }}\right]^{2}
$$

Setting the inner and outer edge of the disc at $r_{i n}=r_{m s}$ and $r_{\text {out }}=\xi r_{m s}$, we have

$$
P_{E M}^{I}\left(a_{*}, \xi\right) / P_{0}=4 \varsigma^{-2} \chi_{m s}^{4}\left(E^{\dagger}-E_{m s}^{\dagger}\right),
$$

where $E^{\dagger}=-u_{0}\left(\xi r_{m s}\right)$ and $E_{m s}^{\dagger}=-u_{0}\left(r_{m s}\right)$ are the specific energies of the disc matter corresponding to $r$ and $r_{m s}$, respectively. ${ }^{[9]}$

We are going to compare our disc power $P_{E M}^{I I}$ with $P_{M H D}$ and $P_{E M}^{I}$. To facilitate comparison between $P_{E M}^{I I}$ and $P_{M H D}$, we take $n=3 / 4$ in Eq.(13), which is the same as given in Eq.(17). By using Eqs.(13), (18) and (20) we have the curves of the three disc powers varying with the radial parameter $\xi$ for the given values of $a_{*}$ as shown in Fig. 3 .

In order to compare the above disc powers more clearly, we define $\eta_{1} \equiv P_{E M}^{I I} / P_{M H D}$ and $\eta_{2} \equiv$ $P_{E M}^{I I} / P_{E M}^{I}$, and have the contours of $\eta_{1}$ and $\eta_{2}$ with different values in $\xi-a_{*}$ parameter spaces as shown in Fig. 4 (a) and (b), respectively.

Inspecting Figs.3 and 4, we have the following results:

(1) If the $\mathrm{BH}$ spin is not extremely high, the three disc powers satisfy the relation, $P_{E M}^{I I}<P_{E M}^{I}<$ $P_{M H D}$. However this order reverses to $P_{M H D}<P_{E M}^{I}<P_{E M}^{I I}$, when the $\mathrm{BH}$ spin approaches unity,

(2) The disc power $P_{E M}^{I I}$ dominates over $P_{E M}^{I}$ and $P_{M H D}$ in two cases: (i) The region is very close to the inner edge of the disc, (ii) The $\mathrm{BH}$ spin is very high.

Recently some authors argued that the BZ power was overestimated, and it is, in general, dominated by the electromagnetic power output of the inner region of the disc, provided that the poloidal magnetic field threading the $\mathrm{BH}$ does not differ significantly in strength from that threading the disc. ${ }^{[14,15]}$ In this Letter we shall discuss the importance of the disc power relative to the BZ power. The BZ power has been derived also based an equivalent circuit in BH magnetosphere in our previous work. ${ }^{[12,13]}$

$$
P_{B Z} / P_{0}=2 a_{*}^{2} \int_{0}^{\theta_{L}} \frac{k(1-k) \sin ^{3} \theta d \theta}{2-(1-q) \sin ^{2} \theta}
$$




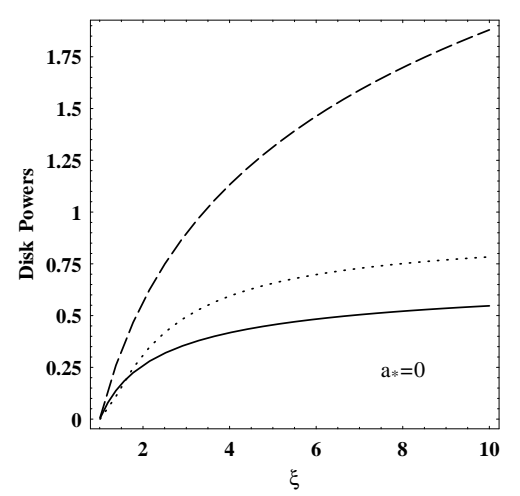

(a)

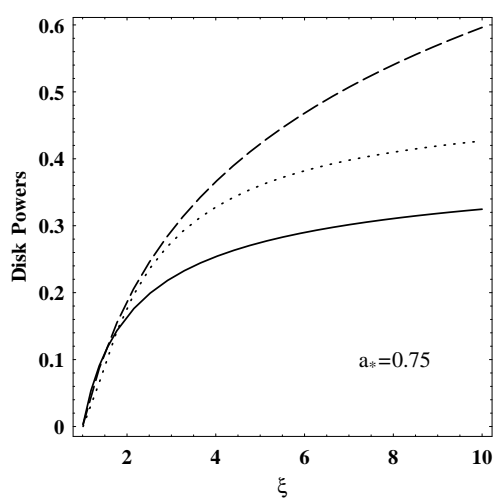

(b)

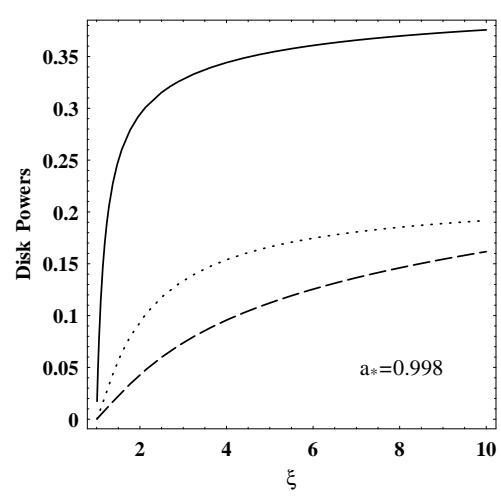

(c)

FIG. 3: The curves of $P_{E M}^{I I}$ (solid line), $P_{M H D}$ (dashed line) and $P_{E M}^{I}$ (dotted line) versus $\xi$ for $1<\xi<10$ with different values of $a_{*}$.

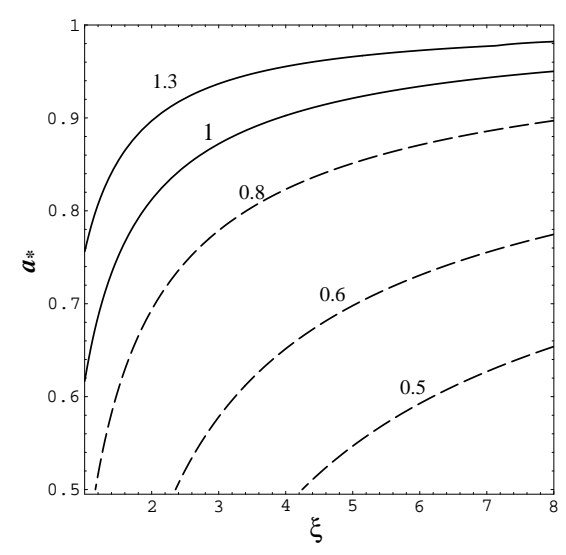

(a)

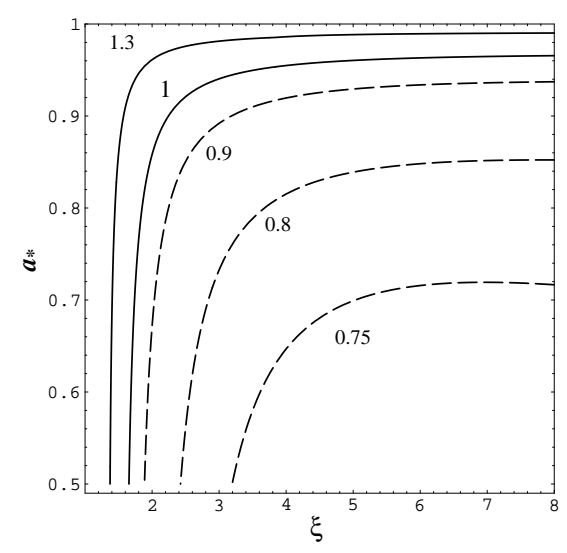

(b)

FIG. 4: The contours of the ratios of the disc powers in $a_{*}-\xi$ parameter space, (a) $\eta_{1} \equiv P_{E M}^{I I} / P_{M H D}$, and (b) $\eta_{2} \equiv P_{E M}^{I I} / P_{E M}^{I}$

where $q \equiv \sqrt{1-a_{*}^{2}}$ is a function of the BH spin, and $k \equiv \Omega_{F} / \Omega_{H}$ is the ratio of the angular velocity of the magnetic field lines to that of the horizon. The angular coordinate $\theta$ varies from 0 to $\theta_{L}$ on the $\mathrm{BH}$ horizon as shown in Fig.1. In calculations $k=0.5$ and $\theta_{L}=0.45 \pi$ are assumed in Eq.(21). In order to compare the disc power with the BZ power clearly, we defined the ratio of the disc power to the BZ power as $\eta=P_{E M}^{I I} / P_{B Z}$. For the given values of power-law index $n$ the contours of $\eta$ are shown in $\xi-a_{*}$ parameter space in Fig.5.

From Fig.5 we find that the disc power will, in general, dominate over the BZ power, except that the $\mathrm{BH}$ spin $a_{*}$ is extremely high. This result is consistent with those given in Refs.[14] and [15].

In our model, different magnetic flux surfaces anchor at different foot point radii $r$ on the disc, which correspond to different light cylinder radii $R_{L}$. It is reasonable that the asymptotic radius of the magnetic flux surface is not greater than its light cylinder radius, i.e., $r^{\prime} \leq R_{L}$. The light cylinder radius satisfy the condition,

$$
R_{L} \Omega_{D}=1
$$

Incorporating Eqs.(7) and (22), we obtain the location of the light cylinder radius of magnetic flux surface as follows, 


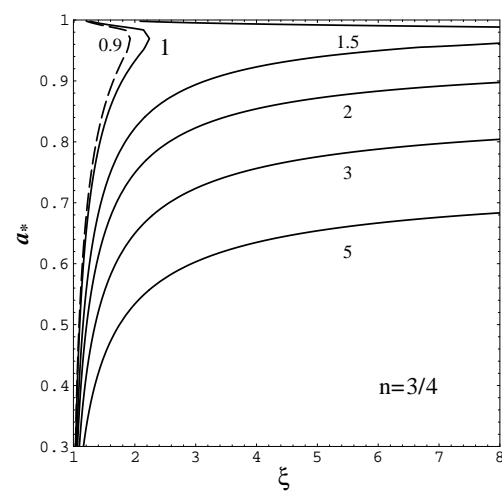

(a)

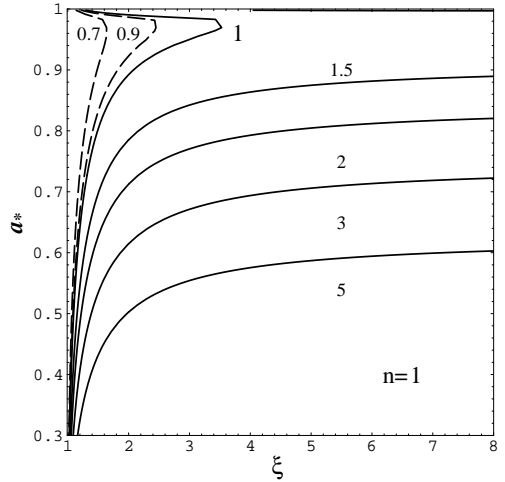

(b)

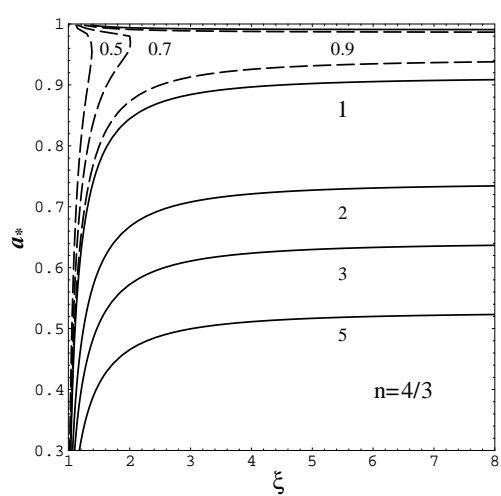

(c)

FIG. 5: The contours of constant values of $\eta$ in $\xi-a_{*}$ parameter space for $n=3 / 4, n=1, n=4 / 3$ in (a), (b) and (c), respectively.

$$
R_{L}=M\left[\left(\sqrt{\xi} \chi_{m s}\right)^{3}+a_{*}\right]
$$

Fendt and Memola defined jet expansion rate as the ratio of the asymptotic jet radius to the foot point jet radius (the 'disc radius'), and the structure of jet is well in accord with the observation of the M87 jet. ${ }^{[10]}$ The observations of the HST show that the radius of the M87 jet is about $100 R_{S},{ }^{[18]}$ where $R_{S}=2 G M / c^{2}$ is Schwarzschild radius. Taking the jet expansion rate $\lambda=10$ as given in Ref.[10], we infer that the foot point jet radius is no greater than $10 R_{S}$.

By using Eq.(13), we have the disc power corresponding to different BH spin $a_{*}$ as listed in Table 1 , where $\xi$ is the upper limit of integration in Eq.(13). In calculations the mass of the central BH is $3 \times 10^{9} M_{\odot}$ as given in Refs.[1] and [2], and the magnetic field on the BH horizon is assumed to be about $3 \times 10^{2}$ gauss as estimated in Ref.[3]. It turns out that the values of the disc power $P_{E M}^{I I}$ in Table 1 are consistent with the value of the M87 jet power. ${ }^{[19]}$

In this Letter the interaction between the $\mathrm{BH}$ and the surrounding disc is neglected for simplification. In fact the magnetic coupling $[12,13,20]$ of the $\mathrm{BH}$ with its surrounding disc will result in a further decrease of the BZ power, and the domination of the disc power over the BZ power will be strengthened in this case. We shall discuss this situation in our future work.

TABLE I: Values of $P_{E M}^{I I}$ in accord with the M87 jet power for different values of the BH $\operatorname{spin} a_{*}$.

\begin{tabular}{llllll}
\hline \hline$a_{*}$ & 0 & 0.3 & 0.5 & 0.75 & 0.998 \\
\hline$r_{m s} / R_{S}$ & 3 & 2.489 & 2.117 & 1.579 & 0.618 \\
\hline$\xi=10 R_{S} / r_{m s}$ & 3.333 & 4.017 & 4.725 & 6.333 & 16.169 \\
\hline$P_{E M}^{I I}\left(\mathrm{erg} \cdot \mathrm{s}^{-1}\right)$ & $2.04 \times 10^{44}$ & $1.91 \times 10^{44}$ & $1.78 \times 10^{44}$ & $1.57 \times 10^{44}$ & $2.06 \times 10^{44}$ \\
\hline
\end{tabular}

\section{References}

[1] Ford H C et al.1994 Astrophys. J. 435 L27

[2] Harms R J et al.1994 Astrophys. J. 435 L35

[3] Blandford R D and Znajek R L 1977 Mon. Not. R. Astron. Soc. 179433

[4] Lovelace R V E 1976 Nature 262 649

[5] Blandford R D and Payne D G 1982 Mon. Not. R. Astron. Soc. 199883

[6] Lovelace R V E et al. 2002 Astrophys. J. 572 L445

[7] Cao X W 2002 Mon. Not. R. Astron. Soc. 332 999

[8] Lee H K 2001 Phys. Rev. D64 043006

[9] Novikov I D and Thorne K S 1973 in Black Holes, ed. Dewitt C Gordon and Breach, New York.

[10] Fendt C and Memola E 2001 Astron.\&Astrophys. 365631 
[11] Blandford R D 1976Mon. Not. R. Astron.Soc.176 465

[12] Wang D X, Xiao K and Lei W H 2002 Mon. Not. R. Astron. Soc. 335655

[13] Wang D X Lei W H and Ma R Y 2003 Mon. Not. R. Astron. Soc. 342851

[14] Livio M, Ogilvie G L and Pringle J E 1999 Astrophys. J. 512 100

[15] Ghosh P and Abramowicz M A 1997 Mon. Not. R. Astron. Soc. 292887

[16] MacDonald D and Thorne K S 1982 Mon. Not. R. Astron. Soc. 198345

[17] Thorne K S, Price R H and Macdonald D A 1986 Black Holes: The Membrane Paradigm, Yale Univ. Press, New Haven

[18] Tsvetanov Z I et al. 1998 Astrophys. J. 493 L83.

[19] Bicknell G V and Begelman M C 1996 Astrophys. J. 467597

[20] Wang D X, Ma R Y, Lei W H and Yao G Z 2003 Astrophys. J. 595109 